\title{
Vague Soft Hypergroups and Vague Soft Hypergroup Homomorphism
}

\author{
Ganeshsree Selvachandran ${ }^{1}$ and Abdul Razak Salleh ${ }^{2}$ \\ ${ }^{1}$ Department of Actuarial Science and Applied Statistics, Faculty of Business and Information Science, UCSI University, \\ Jalan Menara Gading, 56000 Cheras, Kuala Lumpur, Malaysia \\ ${ }^{2}$ School of Mathematical Sciences, Faculty of Science and Technology, Universiti Kebangsaan Malaysia, 43600 Bangi, \\ Selangor DE, Malaysia \\ Correspondence should be addressed to Abdul Razak Salleh; aras@ukm.my
}

Received 28 June 2013; Revised 31 October 2013; Accepted 27 January 2014; Published 31 March 2014

Academic Editor: Marek Reformat

Copyright (c) 2014 G. Selvachandran and A. R. Salleh. This is an open access article distributed under the Creative Commons Attribution License, which permits unrestricted use, distribution, and reproduction in any medium, provided the original work is properly cited.

\begin{abstract}
We introduce and develop the initial theory of vague soft hyperalgebra by introducing the novel concept of vague soft hypergroups, vague soft subhypergroups, and vague soft hypergroup homomorphism. The properties and structural characteristics of these concepts are also studied and discussed.
\end{abstract}

\section{Introduction}

Vague set is a set of objects, each of which has a grade of membership whose value is a continuous subinterval of $[0,1]$. Such a set is characterized by a truth-membership function and a false-membership function. Thus, a vague set is actually a form of fuzzy set, albeit a more accurate form of fuzzy set. Soft set theory has been regarded as an effective mathematical tool to deal with uncertainties. However, it is difficult to be used to represent the vagueness of problem parameters in problem-solving and decision-making contexts. Hence the concept of vague soft sets were introduced as an extension to the notion of soft sets, as a means to overcome the problem of assigning a suitable value for the grade of membership of an element in a set since the exact grade of membership may be unknown. Using the concept of vague soft sets, we are able to ascertain that the grade of membership of an element lies within a certain closed interval.

Hyperstructure theory was first introduced in 1934 by a French mathematician, Marty [1], at the 8th Congress of Scandinavian Mathematicians. In a classical algebraic structure, the composition of two elements is an element, while in an algebraic hyperstructure, the composition of two elements is a set. Since the introduction of the notion of hyperstructures, comprehensive research has been done on this topic and the notions of hypergroupoid, hypergroup, hyperring, and hypermodule have been introduced. A recent book by Corsini and Leoreanu-Fotea [2] expounds on the applications of hyperstructures in the areas of geometry, hypergraphs, binary relations, lattices, fuzzy sets and rough sets, automation, cryptography, combinatorics, codes, artificial intelligence, and probability theory.

The concept of $H_{v}$-structures introduced by Vougiouklis [3] constitute a generalization of the well-known algebraic hyperstructures such as hypergroups, hyperrings, and hypermodules. Some axioms pertaining to the above-mentioned hyperstructures such as the associative law and the distributive law are replaced by their corresponding weak axioms.

The study of fuzzy algebraic structures started with the introduction of the concept of fuzzy subgroup of a group by Rosenfeld [4] in 1971. There is a considerable amount of work that has been done on the connections between fuzzy sets and hyperstructures (see [5]). In 1999, [6] applied the concept of fuzzy sets to the theory of algebraic hyperstructures and defined fuzzy subhypergroup (resp., $H_{v}$-subgroup) of a hypergroup (resp., $H_{v}$-group) which is a generalization of the 
concept of Rosenfeld's fuzzy subgroup of a group. See also Davvaz et al. [7, 8].

The study on soft algebraic hyperstructure theory was initiated by Yamak et al. [9] as an extension to the theory of hyperstructures and soft set theory. They also defined the notion of soft hypergroupoids and soft subhypergroupoids as well as studied some of the basic properties of these concepts. Selvachandran and Salleh [10] on the other hand, introduced the notion of soft hypergroups and soft hypergroup homomorphism.

In this paper, we introduce and develop the initial theory of vague soft hyperalgebra in Rosenfeld's sense. This is done by defining the notion of vague soft hypergroups, vague soft subhypergroups, and vague soft hypergroup homomorphism and using these definitions to develop the initial theory of vague soft hypergroups. The concepts defined in this paper are the combination of the theory of vague soft sets and the theory of fuzzy hypergroups that were introduced by $[6,11]$, respectively. Furthermore, some of the fundamental properties and structural characteristics of these concepts are studied and discussed. Lastly, we prove that there exists a oneto-one correspondence between some of these concepts and their corresponding concepts in soft hypergroup theory as well as classical hypergroup theory.

\section{Preliminaries}

In this section, some well-known and useful definitions pertaining to the theory of soft sets, vague soft sets, hyperstructures, soft hyperstructures, and fuzzy hyperalgebra will be presented. These definitions and concepts will be used throughout this chapter.

Definition 1 (see [12]). A pair $(F, A)$ is called a soft set over $U$, where $F$ is a mapping given by $F: A \rightarrow P(U)$. In other words, a soft set over $U$ is a parameterized family of subsets of the universe $U$. For $\varepsilon \in A, F(\varepsilon)$ may be considered as the set of $\varepsilon$-elements of the soft set $(F, A)$ or as the $\varepsilon$-approximate elements of the soft set.

Example 2 (see [12]). Consider a soft set $(F, E)$ which describes the "attractiveness of houses" that Mr. $X$ is considering for purchase.

Suppose that there are six houses in the universe $U=$ $\left\{h_{1}, h_{2}, h_{3}, h_{4}, h_{5}, h_{6}\right\}$ under consideration, and that $E=$ $\left\{e_{1}, e_{2}, e_{3}, e_{4}, e_{5}, e_{6}\right\}$ is a set of decision parameters. The $e_{i}(i=$ $1,2,3,4,5)$ stand for the parameters "expensive," "beautiful," "wooden," "cheap," and "in green surroundings," respectively.

Consider the mapping $F$ given by "houses $(\cdot)$," where $(\cdot)$ is to be filled in by one of the parameters $e_{i} \in E$. For instance, $F\left(e_{1}\right)$ means "houses (expensive)," and its functional value is the set $\{h \in U: h$ is an expensive house $\}$.

Suppose that $F\left(e_{1}\right)=\left\{h_{2}, h_{4}\right\}, F\left(e_{2}\right)=\left\{h_{1}, h_{3}\right\}, F\left(e_{3}\right)=$ $\emptyset, F\left(e_{4}\right)=\left\{h_{1}, h_{3}, h_{5}\right\}$ and $F\left(e_{5}\right)=\left\{h_{1}\right\}$. Then we can view the soft set $(F, E)$ as consisting of the following collection of approximations:

$(F, E)=\left\{\left(\right.\right.$ expensive houses $\left.\left\{h_{2}, h_{4}\right\}\right)$, (beautiful houses $\left.\left\{h_{1}, h_{3}\right\}\right)$, (wooden houses, $\emptyset$ )(cheap houses $\left\{h_{1}, h_{3}, h_{5}\right\}$ ), (in the green surroundings $\left.\left.\left\{h_{1}\right\}\right)\right\}$.
Each approximation has two parts: a predicate and an approximate value set.

Definition 3 (see [13]). Let $X$ be a space of points (objects) with a generic element of $X$ denoted by $x$. A vague set $V$ in $X$ is characterized by a truth-membership function $t_{V}: X \rightarrow$ $[0,1]$ and a false-membership function $f_{V}: X \rightarrow[0,1]$. The value $t_{V}(x)$ is a lower bound on the grade of membership of $x$ derived from the evidence for $x$ and $f_{V}(x)$ is a lower bound on the negation of $x$ derived from the evidence against $x$. The values $t_{V}(x)$ and $f_{V}(x)$ both associate a real number in the interval $[0,1]$ with each point in $X$, where $t_{V}(x)+f_{V}(x) \leq$ 1. This approach bounds the grade of membership of $x$ to a subinterval $\left[t_{V}(x), 1-f_{V}(x)\right]$ of $[0,1]$. Hence a vague set is a form of fuzzy set, albeit a more accurate form of fuzzy set.

Definition 4 (see [11]). A pair $(\widehat{F}, A)$ is called a vague soft set over $U$ where $\widehat{F}$ is a mapping given by $\widehat{F}: A \rightarrow V(U)$ and $V(U)$ is the power set of vague sets over $U$. In other words, a vague soft set over $U$ is a parametrized family of vague sets of the universe $U$. Every set $\widehat{F}(e)$ for all $e \in A$, from this family may be considered as the set of $e$-approximate elements of the vague soft set $(\widehat{F}, A)$. Hence the vague soft set $(\widehat{F}, A)$ can be viewed as consisting of a collection of approximations of the following form:

$$
\begin{aligned}
(\widehat{F}, A) & =\left\{\widehat{F}\left(x_{i}\right): i=1,2,3, \ldots\right\} \\
& =\left\{\frac{\left[t_{\widehat{F}\left(e_{i}\right)}\left(x_{i}\right), 1-f_{\widehat{F}\left(e_{i}\right)}\left(x_{i}\right)\right]}{x_{i}}: i=1,2,3, \ldots\right\}
\end{aligned}
$$

for all $e \in A$ and for all $x \in U$.

The concept of a support is defined in the literature for both fuzzy sets and formal power series. A similar notion of soft sets was defined by [14].

Example 5 (see [11]). Consider a vague soft set $(\widehat{F}, E)$, where $U$ is a set of six houses under consideration of a decision maker to purchase, which is denoted by $U=$ $\left\{h_{1}, h_{2}, h_{3}, h_{4}, h_{5}, h_{6}\right\}$ and $E$ is a parameter set, where $E=$ $\left\{e_{1}, e_{2}, e_{3}, e_{4}, e_{5}\right\}=\{$ expensive, beautiful, wooden, cheap, in the green surroundings $\}$. The vague soft set $(\widehat{F}, E)$ describes the "attractiveness of the houses" to this decision maker.

Suppose that

$$
\begin{array}{r}
\widehat{F}\left(e_{1}\right)=\left(\frac{[0.1,0.2]}{h_{1}}, \frac{[0.9,1]}{h_{2}}, \frac{[0.3,0.5]}{h_{3}},\right. \\
\left.\frac{[0.8,0.9]}{h_{4}}, \frac{[0.2,0.4]}{h_{5}}, \frac{[0.4,0.6]}{h_{6}}\right), \\
\widehat{F}\left(e_{2}\right)=\left(\frac{[0.9,1]}{h_{1}}, \frac{[0.2,0.7]}{h_{2}}, \frac{[0.6,0.9]}{h_{3}},\right. \\
\left.\frac{[0.2,0.4]}{h_{4}}, \frac{[0.3,0.4]}{h_{5}}, \frac{[0.1,0.6]}{h_{6}}\right),
\end{array}
$$




$$
\begin{gathered}
\widehat{F}\left(e_{3}\right)=\left(\frac{[0,0]}{h_{1}}, \frac{[0,0]}{h_{2}}, \frac{[1,1]}{h_{3}}, \frac{[1,1]}{h_{4}}, \frac{[1,1]}{h_{5}}, \frac{[0,0]}{h_{6}}\right), \\
\widehat{F}\left(e_{4}\right)=\left(\frac{[0.8,0.9]}{h_{1}}, \frac{[0,0.1]}{h_{2}}, \frac{[0.5,0.7]}{h_{3}},\right. \\
\left.\frac{[0.1,0.2]}{h_{4}}, \frac{[0.6,0.8]}{h_{5}}, \frac{[0.4,0.6]}{h_{6}}\right), \\
\widehat{F}\left(e_{5}\right)=\left(\frac{[0.9,1]}{h_{1}}, \frac{[0.2,0.3]}{h_{2}}, \frac{[0.1,0.4]}{h_{3}},\right. \\
\left.\frac{[0.1,0.2]}{h_{4}}, \frac{[0.2,0.4]}{h_{5}}, \frac{[0.7,0.9]}{h_{6}}\right) .
\end{gathered}
$$

The vague soft set $(\widehat{F}, E)$ is a parameterized family $\left\{\widehat{F}\left(e_{i}\right), i=\right.$ $1,2,3,4,5\}$ of vague sets on $U$, and

$$
\begin{aligned}
(\widehat{F}, E)=\{\text { Expensive Houses } \\
=\left(\frac{[0.1,0.2]}{h_{1}}, \frac{[0.9,1]}{h_{2}},\right. \\
\left.\quad \frac{[0.3,0.5]}{h_{3}}, \frac{[0.8,0.9]}{h_{4}}, \frac{[0.2,0.4]}{h_{5}}, \frac{[0.4,0.6]}{h_{6}}\right),
\end{aligned}
$$

Beautiful Houses

$$
\begin{aligned}
&=\left(\frac{[0.9,1]}{h_{1}}, \frac{[0.2,0.7]}{h_{2}}, \frac{[0.6,0.9]}{h_{3}},\right. \\
&\left.\left.\frac{[0.2,0.4]}{h_{4}}, \frac{[0.3,0.4]}{h_{5}}, \frac{[0.1,0.6]}{h_{6}}\right), \ldots\right\} .
\end{aligned}
$$

Definition 6 (see [14]). For a soft set $(F, A)$, the set $\operatorname{Supp}(F, A)=\{x \in A: F(x) \neq \emptyset\}$ is called the support of the soft set $(F, A)$. Thus a null soft set is indeed a soft set with an empty support and a soft set $(F, A)$ is said to be nonnull if $\operatorname{Supp}(F, A) \neq \emptyset$.

Similarly, here we define the notion of the support of a vague soft set $(\widehat{F}, A)$ which is as given below.

Definition 7. Let $(\widehat{F}, A)$ be a vague soft set over $X$. The support of $(\widehat{F}, A)$ denoted by $\operatorname{Supp}(\widehat{F}, A)$ is defined as

$$
\begin{gathered}
\operatorname{Supp}(\widehat{F}, A)=\left\{a \in A: \widehat{F}_{a}(x) \neq \widehat{\emptyset} \text { i.e. } t_{\widehat{F}_{a}}(x) \neq 0,\right. \\
\left.1-f_{\widehat{F}_{a}}(x) \neq 0\right\}
\end{gathered}
$$

for all $x \in X$.

It is to be noted that a null vague soft set is a vague soft set where both the truth and false membership functions are equal to zero. Therefore, a vague soft set $(\widehat{F}, A)$ is said to be nonnull if $\operatorname{Supp}(\widehat{F}, A) \neq \emptyset$.

Definition 8 (see [11]). Let $(\widehat{F}, A)$ and $(\widehat{G}, B)$ be vague soft sets over $U$. Set $(\widehat{F}, A)$ is called a vague soft subset of $(\widehat{G}, B)$ if $A \subseteq B$ and for every $a \in A, \widehat{F}(a)$ and $\widehat{G}(a)$ are the same approximation. In this case, $(\widehat{G}, B)$ is called the vague soft superset of $(\widehat{F}, A)$ and this relationship is denoted as $(\widehat{F}, A) \subseteq$ $(\widehat{G}, B)$.

Definition 9 (see [11]). If $(\widehat{F}, A)$ and $(\widehat{G}, B)$ are two vague soft sets over $U$, the intersection of $(\widehat{F}, A)$ and $(\widehat{G}, B)$, denoted as " $(\widehat{F}, A) \widetilde{\cap}(\widehat{G}, B)$ " is defined by $(\widehat{F}, A) \widetilde{\cap}(\widehat{G}, B)=(\widehat{H}, A \times B)$, where

$$
\begin{array}{r}
t_{\widehat{H}_{c}}(x)= \begin{cases}t_{\widehat{F}_{c}}(x) & c \in A-B, \\
t_{\widehat{G}_{c}}(x) & c \in B-A, \\
\min \left(t_{\widehat{F}_{c}}(x), t_{\widehat{G}_{c}}(x)\right) & c \in B \cap A,\end{cases} \\
1-f_{\widehat{H}_{c}}(x)= \begin{cases}1-f_{\widehat{F}_{c}}(x) & c \in A-B, \\
1-f_{\widehat{G}_{c}}(x) & c \in B-A, \\
\min \left(1-f_{\widehat{F}_{c}}(x), 1-f_{\widehat{G}_{c}}(x)\right) & c \in B \cap A\end{cases}
\end{array}
$$

for all $c \in \operatorname{Supp}(\widehat{H}, C)$ and for all $x \in U$.

Definition 10 (see [11]). If $(\widehat{F}, A)$ and $(\widehat{G}, B)$ are two vague soft sets over $U$, the union of $(\widehat{F}, A)$ and $(\widehat{G}, B)$, denoted by "( $(\widehat{F}, A) \widetilde{\cup}(\widehat{G}, B)$ " is defined by $(\widehat{F}, A) \widetilde{\cup}(\widehat{G}, B)=(\widehat{H}, A \times B)$, where

$$
\begin{array}{r}
t_{\widehat{H}_{c}}(x)= \begin{cases}t_{\widehat{F}_{c}}(x) & c \in A-B, \\
t_{\widehat{G}_{c}}(x) & c \in B-A, \\
\max \left(t_{\widehat{F}_{c}}(x), t_{\widehat{G}_{c}}(x)\right) & c \in B \cap A,\end{cases} \\
1-f_{\widehat{H}_{c}}(x)= \begin{cases}1-f_{\widehat{F}_{c}}(x) & c \in A-B, \\
1-f_{\widehat{G}_{c}}(x) & c \in B-A, \\
\max \left(1-f_{\widehat{F}_{c}}(x), 1-f_{\widehat{G}_{c}}(x)\right) & c \in B \cap A,\end{cases}
\end{array}
$$

for all $c \in \operatorname{Supp}(\widehat{H}, C)$ and for all $x \in U$.

Definition 11 (see [11]). Let $(\widehat{F}, A)$ and $(\widehat{G}, B)$ be vague soft sets over a universe of discourse $X$. The restricted intersection of $(\widehat{F}, A)$ and $(\widehat{G}, B)$, which is denoted by $(\widehat{F}, A) \widetilde{\mathrm{m}}(\widehat{G}, B)=$ $(\widehat{H}, C)$, is a vague soft set $(\widehat{H}, C)$, where $C=A \cap B$ and for all $c \in C$ and $x \in X$,

$$
\begin{gathered}
t_{\widehat{H}_{c}}(x)=\min \left\{t_{\widehat{F}_{c}}(x), t_{\widehat{G}_{c}}(x)\right\}, \\
1-f_{\widehat{H}_{c}}(x)=\min \left\{1-f_{\widehat{F}_{c}}(x), 1-f_{\widehat{G}_{c}}(x)\right\},
\end{gathered}
$$

where $t_{\widehat{H}_{c}}$ and $f_{\widehat{H}_{c}}$ are the truth membership function and false membership function of $\widehat{H}_{c}$. This relationship can be written as $\widehat{H}_{c}(x)=\widehat{F}_{c}(x) \cap \widehat{G}_{c}(x)$.

Definition 12 (see [11]). Let $(\widehat{F}, A)$ and $(\widehat{G}, B)$ be vague soft sets over a universe of discourse $X$. The restricted union of $(\widehat{F}, A)$ and $(\widehat{G}, B)$, which is denoted by $(\widehat{F}, A) \widetilde{\cup}_{\mathscr{R}}(\widehat{G}, B)=(\widehat{H}, C)$, is 
a vague soft set $(\widehat{H}, C)$, where $C=A \cap B$ and for all $c \in C$ and $x \in X$,

$$
\begin{gathered}
t_{\widehat{H}_{c}}(x)=\max \left\{t_{\widehat{F}_{c}}(x), t_{\widehat{G}_{c}}(x)\right\}, \\
1-f_{\widehat{H}_{c}}(x)=\max \left\{1-f_{\widehat{F}_{c}}(x), 1-f_{\widehat{G}_{c}}(x)\right\},
\end{gathered}
$$

where $t_{\widehat{H}_{c}}$ and $f_{\widehat{H}_{c}}$ are the truth membership function and false membership function of $\widehat{H}_{c}$. This relationship can be written as $\widehat{H}_{c}(x)=\widehat{F}_{c}(x) \cup \widehat{G}_{c}(x)$.

Next we present some operations on vague soft sets, namely, the vague soft $(\alpha, \beta)$-cut and vague soft $(\alpha, a)$-cut and the notion of a vague soft characteristic set and vague soft groups.

Definition 13 (see [15]). Let $(\widehat{F}, A)$ be a vague soft set over $U$. Then for all $\alpha, \beta \in[0,1]$, where $\alpha \leq \beta$, the $(\alpha, \beta)$-cut or the vague soft $(\alpha, \beta)$-cut of $(\widehat{F}, A)$, is a subset of $U$ which is as defined below:

$$
\begin{aligned}
&(\widehat{F}, A)_{(\alpha, \beta)}=\left\{x \in U: t_{\widehat{F}_{a}}(x) \geq \alpha,\right. \\
&\left.1-f_{\widehat{F}_{a}}(x) \geq \beta, \text { i.e. } \widehat{F}_{a}(x) \geq[\alpha, \beta]\right\},
\end{aligned}
$$

for all $a \in A$.

If $\alpha=\beta$, then it is called the vague soft $(\alpha, \alpha)$-cut of $(\widehat{F}, A)$ or the $\alpha$-level set of $(\widehat{F}, A)$, denoted by $(\widehat{F}, A)_{(\alpha, \alpha)}$ is a subset of $U$ which is as defined below:

$$
\begin{aligned}
(\widehat{F}, A)_{(\alpha, \alpha)}=\left\{x \in U: t_{\widehat{F}_{a}}(x) \geq \alpha,\right. & \\
& \left.1-f_{\widehat{F}_{a}}(x) \geq \alpha, \text { i.e. } \widehat{F}_{a}(x) \geq[\alpha, \alpha]\right\},
\end{aligned}
$$

for all $a \in A$.

Definition 14 (see [15]). Let $(\widehat{F}, A)$ be a vague soft set over $X$ and $G$ be a nonnull subset of $X$. Then $(\widehat{F}, A)_{G}$ is called a vague soft characteristic set over $G$ in $[0,1]$ and the lower bound and upper bound of $\left(\widehat{F}_{a}\right)_{G}$ are as defined below:

$$
t_{\left(\widehat{F}_{a}\right)_{G}}(x)=1-f_{\left(\widehat{F}_{a}\right)_{G}}(x)= \begin{cases}s & \text { if } x \in G, \\ w & \text { otherwise }\end{cases}
$$

where $\left(\widehat{F}_{a}\right)_{G}$ is a subset of $(\widehat{F}, A)_{G}, x \in X, s, w \in[0,1]$ and $s>w$.

Definition 15 (see [15]). Let $X$ be a group and $(\widehat{F}, A)$ be a vague soft set over $X$. Then $(\widehat{F}, A)$ is called a vague soft group over $X$ if and only if for all $a \in A$ and for every $x, y \in X$, the following conditions are satisfied.

(i) $t_{\widehat{F}_{a}}(x y) \geq \min \left\{t_{\widehat{F}_{a}}(x), t_{\widehat{F}_{a}}(y)\right\}$ and $1-f_{\widehat{F}_{a}}(x y) \geq$ $\min \left\{1-f_{\widehat{F}_{a}}(x), 1-f_{\widehat{F}_{a}}(y)\right\}$, that is $\widehat{F}_{a}(x y) \geq$ $\min \left(\widehat{F}_{a}(x), \widehat{F}_{a}(y)\right)$.

(ii) $t_{\widehat{F}_{a}}\left(x^{-1}\right) \geq t_{\widehat{F}_{a}}(x)$ and $1-f_{\widehat{F}_{a}}\left(x^{-1}\right) \geq 1-f_{\widehat{F}_{a}}(x)$, that is $\widehat{F}_{a}\left(x^{-1}\right) \geq \widehat{F}_{a}(x)$.
TABle 1

\begin{tabular}{ccccc}
\hline$\circ$ & 1 & 2 & 3 & 4 \\
\hline 1 & $\{1,2,3\}$ & $\{1,2\}$ & $\{1,3\}$ & $\{1,3\}$ \\
2 & $\{1,2\}$ & $\{1,2,3\}$ & $\{2,3\}$ & $\{1,3\}$ \\
3 & $\{1,3\}$ & $\{2,3\}$ & $\{1,2,3\}$ & $\{1,2,3\}$ \\
4 & $\{1,3\}$ & $\{2,3\}$ & $\{1,2,3\}$ & $\{1,2,3\}$ \\
\hline
\end{tabular}

That is, for every $a \in A, \widehat{F}_{a}$ is a vague subgroup of $X$ in Rosenfeld's sense.

Theorem 16 (see [15]). Let $(\widehat{F}, A)$ be a vague soft set. Then $(\widehat{F}, A)$ is vague soft group if and only if for every $a \in A$ and $x, y \in X$,

$$
\begin{gathered}
t_{\widehat{F}_{a}}\left(x y^{-1}\right) \geq \min \left\{t_{\widehat{F}_{a}}(x), t_{\widehat{F}_{a}}(y)\right\}, \\
1-f_{\widehat{F}_{a}}\left(x y^{-1}\right) \geq \min \left\{1-f_{\widehat{F}_{a}}(x), 1-f_{\widehat{F}_{a}}(y)\right\},
\end{gathered}
$$

that is,

$$
\widehat{F}_{a}\left(x \cdot y^{-1}\right) \geq \min \left(\widehat{F}_{a}(x), \widehat{F}_{a}(y)\right) .
$$

Definition 17 (see [1]). A hypergroup $\langle H, \circ\rangle$ is a set $H$ equipped with an associative hyperoperation (०) :H×H $\rightarrow$ $P(H)$ which satisfies the reproduction axiom given by $x \circ H=$ $H \circ x=H$ for all $x$ in $H$.

Example 18 (see [9]). Let $H=\{1,2,3,4\}$ be a set with hyperoperation "o" as in Table 1 .

Then $(H, o)$ is a hyperstructure; that is, it is a hypergroup.

Definition 19 (see [16]). A hyperstructure $\langle H, \circ\rangle$ is called a $\mathrm{H}_{v}$-group if the following axioms hold:

(i) $x \circ(y \circ z) \cap(x \circ y) \circ z \neq \emptyset$ for all $x, y, z \in H$,

(ii) $x \circ H=H \circ x=H$ for all $x$ in $H$.

If $\langle H, \circ\rangle$ only satisfies the first axiom, then it is called a $H_{v^{-}}$ semigroup.

Definition 20 (see [1]). A subset $K$ of $H$ is called a subhypergroup if $\langle K, \circ\rangle$ is a hypergroup.

The concepts of hyperstructures were first introduced by Marty as an alternative mathematical tool to complement the concept of ordinary algebraic structures and overcome the shortcomings that is inherent in ordinary algebraic structures. It has been proven to be very useful in various fields. In the context of this paper, these definitions were heavily used to investigate the relationship between the concepts in vague soft hyperalgebraic structures that were introduced in this paper and the corresponding concepts in classical hyperalgebraic theory.

Definition 21 (see [9]). Let $(F, A)$ be a nonnull soft set over $H$. Then $(F, A)$ is called a soft hypergroupoid over $H$ if $F(x)$ is a subhypergroupoid of $H$ for all $x \in \operatorname{Supp}(F, A)$. 
Definition 22 (see [6]). Let $H$ be a hypergroup (or $H_{v}$-group) and let $\mu$ be a fuzzy subset of $H$. Then $\mu$ is said to be a fuzzy subhypergroup (or fuzzy $H_{v}$-subgroup) of $H$ if the following axioms hold:

(i) $\min \{\mu(x), \mu(y)\} \leq \inf \{\mu(z): z \in x \circ y\}$ for all $x$, $y \in H$;

(ii) for all $x, a \in H$, there exists $y \in H$ such that $x \in a \circ y$ and $\min \{\mu(a), \mu(x)\} \leq \mu(y)$;

(iii) for all $x, a \in H$, there exists $z \in H$ such that $x \in z \circ a$ and $\min \{\mu(a), \mu(x)\} \leq \mu(z)$.

The study of fuzzy hyperalgebraic structures was further extended by Leoreanu-Fotea et al. [17] who introduced the notion of fuzzy soft hypergroups and categorized this concept into two classes, namely, fuzzy soft closed hypergroups and fuzzy soft ultraclosed hypergroups. The main definitions of these concepts are as given below.

Let $(H, \circ)$ be a hypergroup which will be denoted simply as $H$ for the sake of simplicity.

Definition 23 (see [17]). Suppose that $(F, A)$ is a fuzzy soft set over $H$. Then $(F, A)$ is called a fuzzy soft hypergroup over $H$ if for all $a \in A$,

(i) For all $x, y \in H, F_{a}(x) \bigwedge F_{a}(y) \leq \bigwedge_{z \in x \circ y} F_{a}(z)$,

(ii) For all $x, y \in H$, there exists $u \in H$ such that $x \in u \circ y$ and $F_{a}(x) \wedge F_{a}(y) \leq F_{a}(u)$,

(iii) For all $x, y \in H$, there exists $v \in H$ such that $x \in y \circ v$ and $F_{a}(x) \wedge F_{a}(y) \leq F_{a}(v)$.

Definition 24 (see [17]). Let $(F, A)$ be a fuzzy soft set over $H$. Then $(F, A)$ is called a fuzzy soft closed hypergroup over $H$ if for all $a \in A$,

(i) for all $x, y \in H, F_{a}(x) \bigwedge F_{a}(y) \leq \bigwedge_{z \in x \circ y} F_{a}(z)$,

(ii) for all $x, y \in H$ and for all $u \in H$, such that $x \in u \circ y$, we have $F_{a}(x) \wedge F_{a}(y) \leq F_{a}(u)$,

(iii) for all $x, y \in H$ and for all $v \in H$, such that $x \in y \circ v$, we have $F_{a}(x) \wedge F_{a}(y) \leq F_{a}(v)$.

Definition 25 (see [17]). Let $(F, A)$ be a fuzzy soft set over $H$. Then $(F, A)$ is called a fuzzy soft ultraclosed hypergroup over $H$ if for all $a \in A$,

(i) for all $x, y \in H, F_{a}(x) \bigwedge F_{a}(y) \leq \bigwedge_{z \in x \circ y} F_{a}(z)$,

(ii) for all $x, y \in H$ and for all $u_{1}, u_{2} \in H$, if $u_{1}, u_{2} \in x-y$, then $F_{a}\left(u_{1}\right)=F_{a}\left(u_{2}\right) \geq F_{a}(x) \bigwedge F_{a}(y)$,

(iii) for all $x, y \in H$ and for all $u_{1}, u_{2} \in H$, if $u_{1}, u_{2} \in y-x$, then $F_{a}\left(u_{1}\right)=F_{a}\left(u_{2}\right) \geq F_{a}(x) \bigwedge F_{a}(y)$.

Beginning with the concept of classical groups, Rosenfeld [4] introduced the concept of a fuzzy subgroup of a group using the concept of fuzzy subsets that were introduced by Zadeh (1965). Early research by Zadeh (1965) and Rosenfeld [4] pertaining to the introduction of the concepts of fuzzy subsets of a set and fuzzy subgroup of a group, respectively, has led to the fuzzification of various algebraic structures (see Akgul, 1988; Bhakat and Das, 1992; Dib, 1994; Davvaz, 1998, 1999, 2009 and Liu, 1982). The notion of fuzzy subgroups of a group has inspired further research and development in the field of fuzzy algebra and subsequently led to the introduction of the notion of fuzzy subhypergroups of a hypergroup by Davvaz [6]. Consequently, research in the area of fuzzy hyperalgebra saw rapid growth and development, mostly using the concept of fuzzy subhypergroups of a hypergroup. In this paper, the Rosenfeld approach is used to introduce the various vague soft hyperalgebraic concepts.

Next, the definition of a fuzzy subgroup of a group that was introduced by Rosenfeld [4] is given.

Definition 26 (see [4]). If $G$ is a classical group with ordinary binary operation $\circ$ and $A$ is a fuzzy subset of $G$, then $A$ is called a fuzzy subgroup of $G$ if for all $x, y \in G$, the following axioms are satisfied:

(i) $A(x \circ y) \geq \min \{A(x), A(y)\}$,

(ii) $A\left(x^{-1}\right) \geq A(x)$.

\section{Vague Soft Hypergroups}

In this section, the concept of vague soft hypergroups in Rosenfeld's sense is introduced. This is an extension to the concept of soft hypergroupoids that were introduced by Yamak et al. [9]. Using this definition, some of the structural characteristics and fundamental properties of vague soft hypergroups are obtained.

From now on, let $\langle H, \circ\rangle$ be a hypergroup, and let $E$ be a set of parameters and $A \subseteq E$. For the sake of simplicity, a hypergroup $\langle H, \circ\rangle$ will be denoted simply as $H$.

Definition 27. Let $(\widehat{F}, A)$ be a nonnull vague soft set over $H$. Then $(\widehat{F}, A)$ is called a vague soft hypergroup over $H$ if the following conditions are satisfied for all $a \in \operatorname{Supp}(\widehat{F}, A)$.

(i) For all $x, y \in H, \min \left\{t_{\widehat{F}_{a}}(x), t_{\widehat{F}_{a}}(y)\right\} \leq \inf \left\{t_{\widehat{F}_{a}}(z)\right.$ : $z \in x \circ y\}$ and $\min \left\{1-f_{\widehat{F}_{a}}(x), 1-f_{\widehat{F}_{a}}(y)\right\} \leq \inf \{1-$ $\left.f_{\widehat{F}_{a}}(z): z \in x \circ y\right\}$, that is, $\min \left\{\widehat{F}_{a}(x), \widehat{F}_{a}(y)\right\} \leq$ $\inf \left\{\widehat{F}_{a}(z): z \in x \circ y\right\}$.

(ii) For all $w, x \in H$, there exists a $y \in H$ such that $x \in w \circ y$ and $\min \left\{t_{\widehat{F}_{a}}(w), t_{\widehat{F}_{a}}(x)\right\} \leq t_{\widehat{F}_{a}}(y)$ and $\min \left\{1-f_{\widehat{F}_{a}}(w), 1-f_{\widehat{F}_{a}}(x)\right\} \leq 1-f_{\widehat{F}_{a}}(y)$, that is $\min \left\{\widehat{F}_{a}(w), \widehat{F}_{a}(x)\right\} \leq \widehat{F}_{a}(y)$

(iii) For all $w, x \in H$, there exists a $z \in H$ such that $x \in z \circ w$ and $\min \left\{t_{\widehat{F}_{a}}(w), t_{\widehat{F}_{a}}(x)\right\} \leq t_{\widehat{F}_{a}}(z)$ and $\min \left\{1-f_{\widehat{F}_{a}}(w), 1-f_{\widehat{F}_{a}}(x)\right\} \leq 1-f_{\widehat{F}_{a}}(z)$, that is $\min \left\{\widehat{F}_{a}(w), \widehat{F}_{a}(x)\right\} \leq \widehat{F}_{a}(z)$.

If $(\widehat{F}, A)$ only satisfies condition (i) of Definition 27 for all $a \in$ $\operatorname{Supp}(\widehat{F}, A)$, then $(\widehat{F}, A)$ is called a vague soft semihypergroup over $H$.

Conditions (ii) and (iii) represent the left and right reproduction axioms, respectively. Then $\widehat{F}_{a}$ is a nonnull vague 
subhypergroup of $H$ (in Rosenfeld's sense) for all $a \in$ $\operatorname{Supp}(\widehat{F}, A)$.

Example 28. Let $H$ be a set, let $\widehat{F}_{a}$ be a subset of $H$ and $(H, \circ)$, and let be a hypergroup with hyperoperation $\circ: \mathrm{H} \times \mathrm{H} \rightarrow$ $P(H)$ which is as defined below.

Let $x, y \in H$. If $\widehat{F}_{a}(x) \geq \widehat{F}_{a}(y)$, then $x \circ y=y \circ x=\{z$ : $\left.z \in H, \widehat{F}_{a}(y) \leq \widehat{F}_{a}(z) \leq \widehat{F}_{a}(x)\right\}$. Next let $(H, *)$ be a group and $(\widehat{F}, A)$ be a vague soft group over $(H, *)$. Consider a nonnull vague soft set $(\widehat{F}, A)$ over $(H, \circ)$ which is as defined below:

$(\widehat{F}, A)^{+}$

$$
=\left\{\left(\widehat{F}_{a}\right)^{+}\left\{x \in H:\left(\widehat{F}_{a}\right)^{+}(x)=\widehat{F}_{a}(x)+1-\widehat{F}_{a}(e)\right\}\right\},
$$

for all $a \in \operatorname{Supp}(\widehat{F}, A)$.

Since $(\widehat{F}, A)$ is a vague soft group over $(H, *)$, then for all $x, y \in(H, *), \widehat{F}_{a}(x y) \geq \min \left\{\widehat{F}_{a}(x), \widehat{F}_{a}(y)\right\}$ and $\widehat{F}_{a}\left(x^{-1}\right) \geq$ $\widehat{F}_{a}(x)$. Then for all $x, y \in(H, \circ)$, we obtain

$$
\begin{aligned}
\inf \left\{\left(\widehat{F}_{a}\right)^{+}(z): z \in x \circ y\right\} \\
\quad=\inf \left\{\left(\widehat{F}_{a}\right)^{+}(z):\left(\widehat{F}_{a}\right)^{+}(y) \leq\left(\widehat{F}_{a}\right)^{+}(z) \leq\left(\widehat{F}_{a}\right)^{+}(x)\right\} \\
\quad=\left(\widehat{F}_{a}\right)^{+}(y)=\widehat{F}_{a}(y)+1-\widehat{F}_{a}(e) \\
\quad \geq \min \left\{\widehat{F}_{a}(x), \widehat{F}_{a}(y)\right\}+1-\widehat{F}_{a}(e) \\
\quad=\min \left\{\widehat{F}_{a}(x)+1-\widehat{F}_{a}(e), \widehat{F}_{a}(y)+1-\widehat{F}_{a}(e)\right\} \\
\quad=\min \left\{\left(\widehat{F}_{a}\right)^{+}(x),\left(\widehat{F}_{a}\right)^{+}(y)\right\} .
\end{aligned}
$$

Therefore we have $\min \left\{\left(\widehat{F}_{a}\right)^{+}(x),\left(\widehat{F}_{a}\right)^{+}(y)\right\} \leq \inf \left\{\left(\widehat{F}_{a}\right)^{+}(z):\right.$ $z \in x \circ y\}$ for all $x, y \in(H, \circ)$. Thus condition (i) of Definition 27 is satisfied. As such, $(\widehat{F}, A)^{+}$is a vague soft semihypergroup over $(H, \circ)$. Next let $w, x \in(H, \circ)$. Consider the two cases given below.

Case 1. $\left(\widehat{F}_{a}\right)^{+}(w) \geq\left(\widehat{F}_{a}\right)^{+}(x)$. Then $\left(\widehat{F}_{a}\right)^{+}(x) \leq\left(\widehat{F}_{a}\right)^{+}(x) \leq$ $\left(\widehat{F}_{a}\right)^{+}(w)$ which implies that $x \in w \circ x$.

Case 2. $\left(\widehat{F}_{a}\right)^{+}(w) \leq\left(\widehat{F}_{a}\right)^{+}(x)$. Then $\left(\widehat{F}_{a}\right)^{+}(w) \leq\left(\widehat{F}_{a}\right)^{+}(x) \leq$ $\left(\widehat{F}_{a}\right)^{+}(x)$ which implies that $x \in x \circ w$.

In both the cases given above, we obtain $x \in w \circ x=$ $x \circ w$. By letting $x=y$, we obtain $x \in w \circ y$ and therefore $\left(\widehat{F}_{a}\right)^{+}(w) \leq\left(\widehat{F}_{a}\right)^{+}(x) \leq\left(\widehat{F}_{a}\right)^{+}(y)$. This implies that $\min \left\{\left(\widehat{F}_{a}\right)^{+}(x),\left(\widehat{F}_{a}\right)^{+}(w)\right\} \leq\left(\widehat{F}_{a}\right)^{+}(y)$. Then for all $w$, $x \in(H, \circ)$, there exists $y \in(H, \circ)$ such that $x \in w \circ y$ and $\min \left\{\left(\widehat{F}_{a}\right)^{+}(x),\left(\widehat{F}_{a}\right)^{+}(w)\right\} \leq\left(\widehat{F}_{a}\right)^{+}(y)$. This means that condition (ii) of Definition 27 is satisfied. Condition (iii) of Definition 27 can be verified in the same manner. Then $\left(\widehat{F}_{a}\right)^{+}$ is a vague subhypergroup of $(H, \circ)$. As such, $(\widehat{F}, A)^{+}$is a vague soft hypergroup over $(H, \circ)$.
Example 29. A vague soft hypergroup $(\widehat{F}, A)$ for which $A$ is a singleton is a vague subhypergroup. Hence vague subhypergroups and also hypergroups are a particular type of vague soft hypergroups.

Definition 30. Let $(F, A)$ be a nonnull soft set over $H$. Then $(F, A)$ is called a soft hypergroup over $H$ if $F(x)$ is a subhypergroup of $H$ for all $x \in \operatorname{Supp}(F, A)$.

Example 31. Let $\widehat{F}_{a}$ be a vague subhypergroup of a hypergroup $(H, \circ)$. This means that $\widehat{F}_{a}$ satisfies the axioms stated in Definition 27. Now consider the family of $\alpha$-level sets for $\widehat{F}_{a}$ given by

$$
\begin{aligned}
(\widehat{F}, A)_{(\alpha, \alpha)}=\left\{x \in H: t_{\widehat{F}_{a}}(x) \geq \alpha,\right. & \\
& \left.1-f_{\widehat{F}_{a}}(x) \geq \alpha, \text { i.e. } \widehat{F}_{a}(x) \geq[\alpha, \alpha]\right\},
\end{aligned}
$$

for all $a \in A$ and $\alpha \in[0,1]$. Then for all $\alpha \in[0,1], \widehat{F}_{a}$ is a subhypergroup of $H$. Hence $(\widehat{F},[0,1])$ is a soft hypergroup over $H$.

Example 32. Any soft hypergroup is a vague subhypergroup since any characteristic function of a subhypergroup is a vague subhypergroup.

Theorem 33. Let $(\widehat{F}, A)$ be a vague soft set over $H$. Then $(\widehat{F}, A)$ is a vague soft hypergroup over $H$ if and only if for all $t \in[0,1]$, $(\widehat{F}, A)_{(t, t)}$ is a soft hypergroup over $H$.

Proof. $(\Rightarrow)$ Let $(\widehat{F}, A)$ be a vague soft hypergroup over $H$. Then for all $a \in \operatorname{Supp}(\widehat{F}, A), \widehat{F}_{a}$ is a nonnull vague subhypergroup of $H$. Now let $x, y \in\left(\widehat{F}_{a}\right)_{(t, t)}$. Thus we obtain the following:

$$
\begin{gathered}
\min \left\{t_{\widehat{F}_{a}}(x), t_{\widehat{F}_{a}}(y)\right\} \geq \min \{t, t\}=t, \\
\min \left\{1-f_{\widehat{F}_{a}}(x), 1-f_{\widehat{F}_{a}}(y)\right\} \geq \min \{t, t\}=t,
\end{gathered}
$$

that is,

$$
\min \left\{\widehat{F}_{a}(x), \widehat{F}_{a}(y)\right\} \geq t
$$

Furthermore, since $\widehat{F}_{a}$ is a vague subhypergroup of $H$, we obtain the following:

$$
\begin{gathered}
\inf \left\{t_{\widehat{F}_{a}}(z): z \in x \circ y\right\} \geq \min \left\{t_{\widehat{F}_{a}}(x), t_{\widehat{F}_{a}}(y)\right\} \geq t, \\
\inf \left\{1-f_{\widehat{F}_{a}}(z): z \in x \circ y\right\} \\
\geq \min \left\{1-f_{\widehat{F}_{a}}(x), 1-f_{\widehat{F}_{a}}(y)\right\} \geq t,
\end{gathered}
$$

which means that $\inf \left\{\widehat{F}_{a}(z): z \in x \circ y\right\} \geq t$. As such, for all $z \in x \circ y$, we obtain $z \in\left(\widehat{F}_{a}\right)_{(t, t)}$ and therefore $x \circ y \subseteq\left(\widehat{F}_{a}\right)_{(t, t)}$. Then for all $z \in\left(\widehat{F}_{a}\right)_{(t, t)}$, we have $z$. $\left(\widehat{F}_{a}\right)_{(t, t)} \subseteq\left(\widehat{F}_{a}\right)_{(t, t)}$. Now let $z, x \in\left(\widehat{F}_{a}\right)_{(t, t)}$. Then there exist $y \in H$ such that $x \in z \circ y$ and $\min \left\{t_{\widehat{F}_{a}}(z), t_{\widehat{F}_{a}}(x)\right\} \leq$ $t_{\widehat{F}_{a}}(y)$ and $\min \left\{1-f_{\widehat{F}_{a}}(z), 1-f_{\widehat{F}_{a}}(x)\right\} \leq 1-f_{\widehat{F}_{a}}(y)$, that is, 
$\min \left\{\widehat{F}_{a}(z), \widehat{F}_{a}(x)\right\} \leq \widehat{F}_{a}(y)$. Since $x, z \in\left(\widehat{F}_{a}\right)_{(t, t)}$, we obtain $\min \left\{t_{\widehat{F}_{a}}(z), t_{\widehat{F}_{a}}(x)\right\} \geq \min \{t, t\}=t$ and $\min \left\{1-f_{\widehat{F}_{a}}(z)\right.$, $\left.1-f_{\widehat{F}_{a}}(x)\right\} \geq \min \{t, t\}=t$, that is, $\min \left\{\widehat{F}_{a}(z), \widehat{F}_{a}(x)\right\} \geq t$. Thus we obtain $t \leq \min \left\{\widehat{F}_{a}(z), \widehat{F}_{a}(x)\right\} \leq \widehat{F}_{a}(y)$ which means that $\widehat{F}_{a}(y) \geq t$ and this implies that $y \in\left(\widehat{F}_{a}\right)_{(t, t)}$. This proves that $\left(\widehat{F}_{a}\right)_{(t, t)} \subseteq z \cdot\left(\widehat{F}_{a}\right)_{(t, t)}$. As such, $z \cdot\left(\widehat{F}_{a}\right)_{(t, t)}=\left(\widehat{F}_{a}\right)_{(t, t)}$ which proves that $\left(\widehat{F}_{a}\right)_{(t, t)}$ is a subhypergroup of $H$. Hence $(\widehat{F}, A)_{(t, t)}$ is a soft hypergroup over $H$.

$(\Leftarrow)$ Assume that $(\widehat{F}, A)_{(t, t)}$ is a soft hypergroup over $H$. Then for all $t \in[0,1],\left(\widehat{F}_{a}\right)_{(t, t)}$ is a nonnull subhypergroup of $H$. For all $x, y \in H$ there are two cases that should be considered.

Case 1. Let $\min \left\{t_{\widehat{F}_{a}}(x), t_{\widehat{F}_{a}}(y)\right\} \leq t_{\widehat{F}_{a}}(x)$ and $\min \left\{1-f_{\widehat{F}_{a}}(x)\right.$, $\left.1-f_{\widehat{F}_{a}}(y)\right\} \leq 1-f_{\widehat{F}_{a}}(x)$, which means that $\min \left\{\widehat{F}_{a}(x)\right.$, $\left.\widehat{F}_{a}(y)\right\} \leq \widehat{F}_{a}(x)$. Next let $\min \left\{\widehat{F}_{a}(x), \widehat{F}_{a}(y)\right\}=t_{0}$. Then $x \in\left(\widehat{F}_{a}\right)_{\left(t_{0}, t_{0}\right)}$ and $y \in\left(\widehat{F}_{a}\right)_{\left(t_{0}, t_{0}\right)}$ and therefore $x \circ y \subseteq$ $\left(\widehat{F}_{a}\right)_{\left(t_{0}, t_{0}\right)}$. Thus for all $z \in x \circ y$, we obtain $\widehat{F}_{a}(z) \geq t_{0}$ which implies that $\min \left\{\widehat{F}_{a}(x), \widehat{F}_{a}(y)\right\} \leq \inf \left\{\widehat{F}_{a}(z): z \in x \circ y\right\}$. As such, condition (i) of Definition 27 is satisfied. To verify condition (ii) of Definition 27, assume that for all $w, x \in H$, $\min \left\{t_{\widehat{F}_{a}}(w), t_{\widehat{F}_{a}}(x)\right\}=t_{1}$ and $\min \left\{1-f_{\widehat{F}_{a}}(w), 1-f_{\widehat{F}_{a}}(x)\right\}=$ $t_{1}$, that is $\min \left\{\widehat{F}_{a}(w), \widehat{F}_{a}(x)\right\}=t_{1}$. Therefore $x \in\left(\widehat{F}_{a}\right)_{\left(t_{1}, t_{1}\right)}$ and $w \in\left(\widehat{F}_{a}\right)_{\left(t_{1}, t_{1}\right)}$. Then there exists $y \in\left(\widehat{F}_{a}\right)_{\left(t_{1}, t_{1}\right)}$ such that $x \in w \circ y$. On the contrary, since $y \in\left(\widehat{F}_{a}\right)_{\left(t_{1}, t_{1}\right)}$, therefore $\widehat{F}_{a}(y) \geq t_{1}$ and thus $\min \left\{\widehat{F}_{a}(w), \widehat{F}_{a}(x)\right\}=t_{1} \leq \widehat{F}_{a}(y)$ which implies that $\min \left\{\widehat{F}_{a}(w), \widehat{F}_{a}(x)\right\} \leq \widehat{F}_{a}(y)$. As such $\widehat{F}_{a}$ is a vague subhypergroup of $H$. Hence $(\widehat{F}, A)$ is a vague soft hypergroup over $H$.

Case 2. Assume that $\min \left\{t_{\widehat{F}_{a}}(x), t_{\widehat{F}_{a}}(y)\right\} \leq t_{\widehat{F}_{a}}(y)$ and $\min \left\{1-f_{\widehat{F}_{a}}(x), 1-f_{\widehat{F}_{a}}(y)\right\} \leq 1-f_{\widehat{F}_{a}}(y)$, which means that $\min \left\{\widehat{F}_{a}(x), \widehat{F}_{a}(y)\right\} \leq \widehat{F}_{a}(y)$. The subsequent proof is similar to that of the proof for Case 1 and in a similar manner it can be proven that $\widehat{F}_{a}$ is a vague subhypergroup of $H$.

In both the cases, it has been proven that $\widehat{F}_{a}$ is a vague subhypergroup of $H$ and therefore it is proven that $(\widehat{F}, A)$ is a vague soft hypergroup over $H$.

Theorem 33 proves that there exists a one-to-one correspondence between vague soft hypergroups and soft hypergroups of a hypergroup.

Corollary 34. Let $(\widehat{F}, A)$ be a vague soft hypergroup over $H$. If $0 \leq t_{1}<t_{2} \leq 1$, then $\left(\widehat{F}_{a}\right)_{\left(t_{1}, t_{1}\right)}=\left(\widehat{F}_{a}\right)_{\left(t_{2}, t_{2}\right)}$ if and only if there does not exist $x \in H$ such that $t_{1} \leq \widehat{F}_{a}(x)<t_{2}$.

Proof. $(\Rightarrow)$ Let $0 \leq t_{1}<t_{2} \leq 1$ and $\left(\widehat{F}_{a}\right)_{\left(t_{1}, t_{1}\right)}=\left(\widehat{F}_{a}\right)_{\left(t_{2}, t_{2}\right)}$. Then there exists $x \in H$ such that $\widehat{F}_{a}(x) \geq t_{1}$ and $\widehat{F}_{a}(x) \geq t_{2}$. Since $\left(\widehat{F}_{a}\right)_{\left(t_{1}, t_{1}\right)}=\left(\widehat{F}_{a}\right)_{\left(t_{2}, t_{2}\right)}$, we obtain $t_{1}=t_{2} \leq \widehat{F}_{a}(x)$. Therefore, there does not exist $x \in H$ such that $t_{1} \leq \widehat{F}_{a}(x)<t_{2}$.

$(\Leftarrow)$ Assume that there does not exist $x \in H$ such that $t_{1} \leq \widehat{F}_{a}(x)<t_{2}$. From $t_{1} \leq \widehat{F}_{a}(x)<t_{2}$, we obtain $\widehat{F}_{a}(x) \geq$ $t_{1}$ and $\widehat{F}_{a}(x)<t_{2}$, that is, $\widehat{F}_{a}(x) \nsupseteq t_{2}$. However, this is not possible since we have $t_{1}<t_{2}$ because of $0 \leq t_{1}<t_{2} \leq 1$. As such, since $\widehat{F}_{a}(x) \geq t_{1}$, then $\widehat{F}_{a}(x) \geq t_{2}$ too for all $x \in H$. Thus $x \in\left(\widehat{F}_{a}\right)_{\left(t_{1}, t_{1}\right)}$ and $x \in\left(\widehat{F}_{a}\right)_{\left(t_{2}, t_{2}\right)}$. For $x \in H$ to satisfy both the conditions $\widehat{F}_{a}(x) \geq t_{1}$ and $\widehat{F}_{a}(x) \geq t_{2}, \widehat{F}_{a}(x)$ must satisfy the condition $\widehat{F}_{a}(x)=t_{1}$ and $\widehat{F}_{a}(x)=t_{2}$. This implies that $t_{1}=t_{2}$. Therefore it has been proven that $\left(\widehat{F}_{a}\right)_{\left(t_{1}, t_{1}\right)}=\left(\widehat{F}_{a}\right)_{\left(t_{2}, t_{2}\right)}$.

Definition 35 (see [6]). Let $H$ be a set and $\widehat{F}_{a}$ be a subset of $H$. The hyperoperation $\circ: H \times H \rightarrow P(H)$ is as defined below.

Let $x, y \in H$. If $\widehat{F}_{a}(x) \leq \widehat{F}_{a}(y)$, then $x \circ y=y \circ x=\{t$ : $\left.t \in H, \widehat{F}_{a}(x) \leq \widehat{F}_{a}(t) \leq \widehat{F}_{a}(y)\right\}$.

Proposition 36. Let $(H, *)$ be a group. Then $(\widehat{F}, A)$ is a vague soft group over $(H, *)$ if and only if $(\widehat{F}, A)$ is a soft hypergroup over $(H, \circ)$.

Proof. $(\Rightarrow)$ Let $(\widehat{F}, A)$ be a vague soft group over group $(H, *)$ and $(H, \circ)$ be a hypergroup with hyperoperation as defined in Definition 35. Then for all $x, y \in(H, *), \widehat{F}_{a}(x y) \geq$ $\min \left\{\widehat{F}_{a}(x), \widehat{F}_{a}(y)\right\}$ and $\widehat{F}_{a}\left(x^{-1}\right) \geq \widehat{F}_{a}(x)$. Therefore for all $x$, $y \in(H, *)$, we obtain the following:

$$
\begin{aligned}
\inf & \left\{\widehat{F}_{a}(z): z \in x \circ y\right\} \\
& =\inf \left\{\widehat{F}_{a}(z): \widehat{F}_{a}(x) \leq \widehat{F}_{a}(z) \leq \widehat{F}_{a}(y)\right\} \\
& =\widehat{F}_{a}(x) \geq \min \left\{\widehat{F}_{a}(x), \widehat{F}_{a}(y)\right\} .
\end{aligned}
$$

Hence, condition (i) of Definition 27 has been verified. Now let $w, x \in H$. Therefore if $\widehat{F}_{a}(w) \leq \widehat{F}_{a}(x)$, then $\widehat{F}_{a}(w) \leq$ $\widehat{F}_{a}(x) \leq \widehat{F}_{a}(x)$, which implies that $x \in w \circ x$ (from Definition 35) and if $\widehat{F}_{a}(x) \leq \widehat{F}_{a}(w)$, then $\widehat{F}_{a}(x) \leq \widehat{F}_{a}(x) \leq$ $\widehat{F}_{a}(w)$ which also implies that $x \in x \circ w$. Thus we obtain $x \in x \circ a=a \circ x$. As such, if we let $y=x$, then for any case we obtain $\min \left\{\widehat{F}_{a}(x), \widehat{F}_{a}(w)\right\} \leq \widehat{F}_{a}(y)$. Hence condition (ii) of Definition 27 has been verified. Then $\widehat{F}_{a}$ is a subhypergroup of $(H, \circ)$. As such, it has been proven that $(\widehat{F}, A)$ is a soft hypergroup over $(H, \circ)$.

$(\Leftarrow)$ Let $(\widehat{F}, A)$ be a soft hypergroup over $(H, \circ)$ and let $x$, $y \in(H, \circ)$. We consider two cases.

Case 1. Suppose that $\widehat{F}_{a}(x) \leq \widehat{F}_{a}\left(y^{-1}\right)$. Then we obtain the following:

$$
\begin{aligned}
\inf & \left\{\widehat{F}_{a}(z): z \in x * y^{-1}\right\} \\
& =\inf \left\{\widehat{F}_{a}(z): \widehat{F}_{a}(x) \leq \widehat{F}_{a}(z) \leq \widehat{F}_{a}\left(y^{-1}\right)\right\} \\
& =\widehat{F}_{a}(x) \geq \min \left\{\widehat{F}_{a}(x), \widehat{F}_{a}\left(y^{-1}\right)\right\} .
\end{aligned}
$$

Therefore, $\widehat{F}_{a}$ is a vague subgroup of $(H, *)$. 
Case 2. Suppose that $\widehat{F}_{a}\left(y^{-1}\right) \leq \widehat{F}_{a}(x)$. Then we obtain the following:

$$
\begin{aligned}
\inf \left\{\widehat{F}_{a}(z): z \in x * y^{-1}\right\} \\
\quad=\inf \left\{\widehat{F}_{a}(z): \widehat{F}_{a}\left(y^{-1}\right) \leq \widehat{F}_{a}(z) \leq \widehat{F}_{a}(x)\right\} \\
\quad=\widehat{F}_{a}\left(y^{-1}\right) \geq \min \left\{\widehat{F}_{a}(x), \widehat{F}_{a}\left(y^{-1}\right)\right\} .
\end{aligned}
$$

Therefore, $\widehat{F}_{a}$ is a vague subgroup of $(H, *)$. In both the cases, it has been proven that $\widehat{F}_{a}$ is a vague subgroup of $(H, *)$. Hence $(\widehat{F}, A)$ is a vague soft group over $(H, *)$.

Theorem 37. Let $(\widehat{F}, A)$ be a vague soft set over $H$. Then $(\widehat{F}, A)$ is a vague soft hypergroup over $H$ if and only if for all $\alpha, \beta \in$ $[0,1],(\widehat{F}, A)_{(\alpha, \beta)}$ is a subhypergroup of $H$.

Proof. The proof is straightforward.

As a result of Theorem 37, we obtain Corollary 38 which is as given below.

Corollary 38. Let $(\widehat{F}, A)$ be a vague soft set over $H$. Then $(\widehat{F}, A)$ is a vague soft hypergroup over $H$ if and only if for all $\alpha \in[0,1],(\widehat{F}, A)_{(\alpha, \alpha)}$ is a subhypergroup of $H$.

Theorem 37 and Corollary 38 prove that there exists a one-to-one correspondence between vague soft hypergroups and subhypergroups of a hypergroup.

Theorem 39. Let $K$ be a non-null subset of $H$ and $(\widehat{F}, A)_{K}$ be a vague soft characteristic set over $K$. If $(\widehat{F}, A)$ is a vague soft hypergroup over $H$, then $K$ is a subhypergroup of $H$.

Proof. The proof is straightforward.

Theorem 40. Let $(\widehat{F}, A)$ and $(\widehat{G}, B)$ be vague soft hypergroups over $H$. Then $(\widehat{F}, A) \widetilde{\cap}(\widehat{G}, B)$ is a vague soft hypergroup over $H$ if it is nonnull.

Proof. The proof can be easily obtained using Definition 9.

Remarks/Note. Theorem 40 also holds true for other operations in vague soft set theory such as union, restricted intersection, restricted union, "AND," and “OR”.

\section{Vague Soft Hypergroup Homomorphism}

In the first part of this section, the notion of vague soft function as well as the image and preimage of a vague soft set under a vague soft function are introduced and defined. These definitions will then be used in the context of vague soft hypergroups to introduce the notion of vague soft hypergroup homomorphism. Lastly, it is proven that the vague soft hypergroup homomorphism preserves vague soft hypergroups.
Definition 41. Let $\varphi: X=\rightarrow Y$ and $\psi: A \rightarrow B$ be two functions, where $A$ and $B$ are parameter sets for the classical sets $X$ and $Y$, respectively. Let $(\widehat{F}, A)$ and $(\widehat{G}, B)$ be vague soft sets over $X$ and $Y$, respectively. Then the ordered pair $(\varphi, \psi)$ is called a vague soft function from $(\widehat{F}, A)$ to $(\widehat{G}, B)$, and it is denoted as $(\varphi, \psi):(\widehat{F}, A) \rightarrow(\widehat{G}, B)$.

Definition 42. Let $(\widehat{F}, A)$ and $(\widehat{G}, B)$ be vague soft sets over $X$ and $Y$, respectively. Let $(\varphi, \psi):(\widehat{F}, A) \rightarrow(\widehat{G}, B)$ be a vague soft function.

(i) The image of $(\widehat{F}, A)$ under the vague soft function $(\varphi, \psi)$, which is denoted as $(\varphi, \psi)(\widehat{F}, A)$, is a vague soft set over $Y$, which is defined as

$$
(\varphi, \psi)(\widehat{F}, A)=(\varphi(\widehat{F}), \psi(A)),
$$

where

$$
\varphi\left(\widehat{F}_{a}\right)(\varphi(x))=(\varphi(\widehat{F}))_{\psi(a)}(y)
$$

for all $a \in A, x \in X$, and $y \in Y$.

(ii) The preimage of $(\widehat{G}, B)$ under the vague soft function $(\varphi, \psi)$, which is denoted as $(\varphi, \psi)^{-1}(\widehat{G}, B)$, is a vague soft set over $X$, which is defined as

$$
(\varphi, \psi)^{-1}(\widehat{G}, B)=\left(\varphi^{-1}(\widehat{G}), \psi^{-1}(B)\right),
$$

where

$$
\varphi^{-1}\left(\widehat{G}_{b}\right)\left(\varphi^{-1}(y)\right)=\left(\varphi^{-1}(\widehat{G})\right)_{\psi^{-1}(b)}(x)
$$

for all $b \in B, x \in X$, and $y \in Y$.

If $\varphi$ and $\psi$ are injective (surjective), then the vague soft function $(\varphi, \psi)$ is said to be injective (surjective).

Definition 43 (see [18]). Let $\left(H_{1}, \circ\right)$ and $\left(H_{2}, *\right)$ be hypergroups. The map $f: H_{1} \rightarrow H_{2}$ is called a good homomorphism if $f(x \circ y)=f(x) * f(y)$ and it is called an inclusion homomorphism if $f(x \circ y) \subseteq f(x) * f(y)$ for all $x$, $y \in H_{1}$.

Definition 44. Let $\left(H_{1}, \circ\right)$ and $\left(H_{2}, *\right)$ be hypergroups and $(\widehat{F}, A)$ and $(\widehat{G}, B)$ be vague soft hypergroups over $H_{1}$ and $H_{2}$, respectively. Let $(\varphi, \psi):(\widehat{F}, A) \rightarrow(\widehat{G}, B)$ be a vague soft function. Then we obtain the following.

(a) $(\varphi, \psi)$ is called a vague soft inclusion hypergroup homomorphism if the following conditions are satisfied:

(i) $\varphi: H_{1} \rightarrow H_{2}$ is an inclusion homomorphism,

(ii) $\psi: A \rightarrow B$ is a function,

(iii) $\varphi(\widehat{F}(x))=\widehat{G}(\psi(x))$ for all $x \in \operatorname{Supp}(\widehat{F}, A)$.

(b) $(\varphi, \psi)$ is called a vague soft good hypergroup homomorphism if the following conditions are satisfied:

(i) $\varphi: H_{1} \rightarrow H_{2}$ is a good homomorphism, 
(ii) $\psi: A \rightarrow B$ is a function,

(iii) $\varphi(\widehat{F}(x))=\widehat{G}(\psi(x))$ for all $x \in \operatorname{Supp}(\widehat{F}, A)$.

Theorem 45. Let $(\widehat{F}, A)$ and $(\widehat{G}, B)$ be vague soft hypergroups over $\left(H_{1}, \circ\right)$ and $\left(H_{2}, *\right),(\varphi, \psi):(\widehat{F}, A) \rightarrow(\widehat{G}, B)$ be a vague soft good hypergroup homomorphism and $\psi: A \rightarrow$ $B$ be a bijective mapping. Then $(\varphi(\widehat{F}), \psi(A))$ is a vague soft hypergroup over $\left(\mathrm{H}_{2}, *\right)$.

Proof. Since $(\varphi, \psi)$ is a vague soft good hypergroup homomorphism, it can be observed that $\operatorname{Supp}(\varphi(\widehat{F}), \psi(A))=$ $\psi(\operatorname{Supp}(\widehat{F}, A))$. Next let $w_{1}, w_{2} \in H_{2}$. Then there exist $x$, $y \in H_{1}$ such that $\varphi(x)=w_{1}$ and $\varphi(y)=w_{2}$. Therefore for all $x, y \in H_{1}$ and for all $a \in \operatorname{Supp}(\widehat{F}, A)$,

$$
\begin{aligned}
\min & \left\{\varphi\left(\widehat{F}_{a}\right)(\varphi(x)), \varphi\left(\widehat{F}_{a}\right)(\varphi(y))\right\} \\
& =\min \left\{(\varphi(\widehat{F}))_{\psi(a)}\left(w_{1}\right),(\varphi(\widehat{F}))_{\psi(a)}\left(w_{2}\right)\right\} \\
& \leq \inf \left\{(\varphi(\widehat{F}))_{\psi(a)}(u): u \in w_{1} * w_{2}\right\} \\
& =\inf \left\{\varphi\left(\widehat{F}_{a}\right)(\varphi(z))\right\},
\end{aligned}
$$

where $z \in H_{1}$ such that $\varphi(z) \in \varphi(x) * \varphi(y)$ and $\varphi(z)=u$.

Furthermore for all $w, x \in H_{1}$, there exist $u_{1}, u_{2} \in H_{2}$ such that $\varphi(w)=u_{1}$ and $\varphi(x)=u_{2}$. As such, for all $w, x \in H_{1}$, and $a \in \operatorname{Supp}(\widehat{F}, A)$ it follows that

$$
\begin{aligned}
\min & \left\{\varphi\left(\widehat{F}_{a}\right)(\varphi(w)), \varphi\left(\widehat{F}_{a}\right)(\varphi(x))\right\} \\
& =\min \left\{(\varphi(\widehat{F}))_{\psi(a)}\left(u_{1}\right),(\varphi(\widehat{F}))_{\psi(a)}\left(u_{2}\right)\right\} \\
& \leq \inf \left\{(\varphi(\widehat{F}))_{\psi(a)}(b): u_{2} \in u_{1} * b\right\} \\
& =\inf \left\{\varphi\left(\widehat{F}_{a}\right)(\varphi(y))\right\},
\end{aligned}
$$

where $y \in H_{1}$ such that $\varphi(x) \in \varphi(w) * \varphi(y)$ and $\varphi(y)=b$ and also

$$
\begin{aligned}
\min & \left\{\varphi\left(\widehat{F}_{a}\right)(\varphi(w)), \varphi\left(\widehat{F}_{a}\right)(\varphi(x))\right\} \\
& =\min \left\{(\varphi(\widehat{F}))_{\psi(a)}\left(u_{1}\right),(\varphi(\widehat{F}))_{\psi(a)}\left(u_{2}\right)\right\} \\
& \leq \inf \left\{(\varphi(\widehat{F}))_{\psi(a)}(c): u_{2} \in c * u_{1}\right\} \\
& =\inf \left\{\varphi\left(\widehat{F}_{a}\right)(\varphi(z))\right\},
\end{aligned}
$$

where $z \in H_{1}$ such that $\varphi(x) \in \varphi(z) * \varphi(w)$ and $\varphi(z)=c$.

Hence $(\varphi(\widehat{F}), \psi(A))$ is a vague soft hypergroup over $\left(H_{2}, *\right)$ by Definition 27.

Theorem 46. Let $(\widehat{F}, A)$ and $(\widehat{G}, B)$ be vague soft hypergroups over $\left(H_{1}, \circ\right)$ and $\left(H_{2}, *\right)$, respectively, and $(\varphi, \psi):(\widehat{F}, A) \rightarrow$ $(\widehat{G}, B)$ be a vague soft inclusion hypergroup homomorphism. Then $\left(\varphi^{-1}(\widehat{G}), \psi^{-1}(B)\right)$ is a vague soft hypergroup over $\left(H_{1}, \circ\right)$.

Proof. The proof is similar to that of Theorem 45.
Theorems 45 and 46 prove that the homomorphic image and preimage of a vague soft hypergroup are also vague soft hypergroups.

Theorem 47. Let $H_{1}, H_{2}$, and $H_{3}$ be nonnull hypergroups and $(\widehat{F}, A),(\widehat{G}, B)$, and $(\widehat{J}, C)$ be vague soft hypergroups over $H_{1}, H_{2}$, and $H_{3}$, respectively. Let $(\varphi, \psi):(\widehat{F}, A) \rightarrow$ $(\widehat{G}, B)$ and $\left(\varphi^{\prime}, \psi^{\prime}\right):(\widehat{G}, B) \rightarrow(\widehat{J}, C)$ be vague soft good (inclusion) hypergroup homomorphisms. Then $\left(\varphi^{\prime} \circ \varphi, \psi^{\prime} \circ \psi\right)$ : $(\widehat{F}, A) \rightarrow(\widehat{J}, C)$ is a vague soft good (inclusion) hypergroup homomorphism.

Proof. The proof is straightforward.

\section{Vague Soft Subhypergroups}

In this section, the concept of vague soft subhypergroups is introduced. This concept is an extension of the concept of vague soft hypergroups that was introduced in Section 3. Some of the basic properties of this concept are then studied and discussed.

Definition 48. Let $(\widehat{F}, A)$ and $(\widehat{G}, B)$ be vague soft hypergroups over $H$. Then $(\widehat{F}, A)$ is called a vague soft subhypergroup of $(\widehat{G}, B)$, which is denoted as $(\widehat{F}, A) \leq_{S}(\widehat{G}, B)$, if the following conditions are satisfied:

(i) $A \subseteq B$,

(ii) $\widehat{F}_{a}$ is a non-null vague subhypergroup of $\widehat{G}_{a}$ for all $a \epsilon$ $\operatorname{Supp}(\widehat{F}, A)$.

Theorem 49. Let $(\widehat{F}, A)$ and $(\widehat{G}, B)$ be vague soft hypergroups over $H$ and $(\widehat{F}, A)$ be a vague soft subset of $(\widehat{G}, B)$. Then $(\widehat{F}, A) \leq_{S}(\widehat{G}, B)$.

Proof. Let $(\widehat{F}, A)$ and $(\widehat{G}, B)$ be vague soft hypergroups over $H$ and $(\widehat{F}, A)$ be a vague soft subset of $(\widehat{G}, B)$, that is, $(\widehat{F}, A) \subseteq$ $(\widehat{G}, B)$. Since $(\widehat{F}, A) \subseteq(\widehat{G}, B)$, by Definition 6 , it can be concluded that $A \subseteq B$. Furthermore, since $(\widehat{F}, A)$ and $(\widehat{G}, B)$ are vague soft hypergroups over $H$ and $(\widehat{F}, A) \subseteq(\widehat{G}, B)$, $\widehat{F}_{a}$ is a nonnull vague subhypergroup of $\widehat{G}_{a}$ for all $a \epsilon$ $\operatorname{Supp}(\widehat{F}, A)$. As such, $(\widehat{F}, A)$ is a vague soft hypergroup over $(\widehat{G}, B)$. Hence $(\widehat{F}, A)$ is a vague soft subhypergroup of $(\widehat{G}, B)$, that is, $(\widehat{F}, A) \leq_{S}(\widehat{G}, B)$.

Theorem 50. Let $(\widehat{F}, A)$ and $(\widehat{G}, B)$ be vague soft hypergroups over $H$ and $(\widehat{F}, A)$ be a vague soft subhypergroup of $(\widehat{G}, B)$. Then

(i) $(\widehat{F}, A) \widetilde{\mathrm{n}}(\widehat{G}, B)$ is a vague soft subhypergroup of $(\widehat{G}, B)$.

(ii) $(\widehat{F}, A) \widetilde{U}_{\mathscr{R}}(\widehat{G}, B)$ is a vague soft subhypergroup of $(\widehat{G}, B)$.

Proof. (i) The proof is similar to that of Theorem 40 and can be obtained using Definition 9.

(ii) The proof is straightforward using Definition 10. 
Theorem 51. Let $\varphi: H_{1} \rightarrow H_{2}$ be a good homomorphism for hypergroups.

(i) Let $(\widehat{F}, A)$ and $(\widehat{G}, B)$ be vague soft hypergroups over $H_{1}$. If $(\widehat{F}, A) \leq_{S}(\widehat{G}, B)$, then $(\varphi(\widehat{F}), A) \leq_{S}(\varphi(\widehat{G}), B)$.

(ii) Let $(\widehat{F}, A)$ and $(\widehat{G}, B)$ be vague soft hypergroups over $H_{2}$. If $(\widehat{F}, A) \leq_{S}(\widehat{G}, B)$, then $\left(\varphi^{-1}(\widehat{F}), A\right) \leq_{S}$ $\left(\varphi^{-1}(\widehat{G}), B\right)$.

Proof. The proof is easily obtained using Definition 48 .

\section{Conclusion}

In this paper, we presented an initial study on the theory of vague soft hyperalgebra. We introduced the novel concepts of vague soft hypergroups and vague soft subhypergroups and proceeded to study and discuss some of the fundamental properties and structural characteristics of these concepts. Furthermore, we also proved that there exists a one-to-one correspondence between some of the concepts introduced in this paper and the corresponding concepts in soft hypergroup theory and classical hypergroup theory. We also introduced the notion of vague soft hypergroup homomorphism using the concepts of vague soft functions as well as the image and preimage of a vague soft set under a vague soft function. Lastly, it was proved that the homomorphic image and pre-image of vague soft hypergroups are also vague soft hypergroups, which implies that the vague soft hypergroup homomorphism preserves vague soft hypergroups. It is to be noted that this is among the initial research in the area of vague soft hyperalgebra and hence it serves as a first step in the advancement and development of the theory of vague soft sets as well as the theory of vague soft hyperalgebra. We are currently developing the theory of vague soft hyperalgebra for other hyperalgebraic structures.

\section{Conflict of Interests}

The authors declare that there is no conflict of interests regarding the publication of this paper.

\section{Acknowledgments}

The authors would like to extend their sincere gratitude to the reviewers and the editor in charge for their constructive comments and suggestions which have helped to improve the quality of this paper. The authors would like to gratefully acknowledge the financial support received from the Faculty of Science and Technology, Universiti Kebangsaan Malaysia (UKM), under Grant no. AP-2013-009.

\section{References}

[1] F. Marty, "Sur une generalization de la notion de group," in Proceedings of the 8th Congress des Mathematiciens Scandinaves, pp. 45-49, Stockholm, Sweden, 1934.
[2] P. Corsini and V. Leoreanu-Fotea, Application of Hyperstructures Theory: Advances in Mathematics, Kluwer Academic, Dodrecht, The Netherlands, 2003.

[3] T. Vougiouklis, " $H_{v}$-groups defined on the same set," Discrete Mathematics, vol. 155, no. 1-3, pp. 259-265, 1996.

[4] A. Rosenfeld, "Fuzzy groups," Journal of Mathematical Analysis and Applications, vol. 35, no. 3, pp. 512-517, 1971.

[5] B. Davvaz, "On $H_{v}$-rings and Fuzzy $H_{v}$-ideal," Journal of Fuzzy Mathematics, vol. 6, no. 1, pp. 33-42, 1998.

[6] B. Davvaz, "Fuzzy $H_{v}$-groups," Fuzzy Sets and Systems, vol. 101, no. 1, pp. 191-195, 1999.

[7] B. Davvaz, M. Fathi, and A. R. Salleh, "Fuzzy hypergroups based on fuzzy spaces," Annals of Fuzzy Mathematics and Informatics, vol. 6, no. 3, pp. 767-779, 2013.

[8] B. Davvaz, M. Fathi, and A. R. Salleh, "Fuzzy hyperrings $\left(H_{v^{-}}\right.$ rings) based on fuzzy universal sets," Information Sciences, vol. 180, pp. 3021-3032, 2010.

[9] S. Yamak, O. Kazanci, and B. Davvaz, "Soft hyperstructure," Computers and Mathematics with Applications, vol. 62, no. 2, pp. 797-803, 2011.

[10] G. Selvachandran and A. R. Salleh, "Soft hypergroups and soft hypergroup homomorphism," in Proceedings of the 20th National Symposium on Mathematical Sciences, vol. 1522 of AIP Conference Proceedings, pp. 821-827, 2013.

[11] W. Xu, J. Ma, S. Wang, and G. Hao, "Vague soft sets and their properties," Computers and Mathematics with Applications, vol. 59, no. 2, pp. 787-794, 2010.

[12] D. Molodtsov, "Soft set theory-first results," Computers and Mathematics with Applications, vol. 37, no. 4-5, pp. 19-31, 1999.

[13] W.-L. Gau and D. J. Buehrer, "Vague sets," IEEE Transactions on Systems, Man and Cybernetics, vol. 23, no. 2, pp. 610-614, 1993.

[14] F. Feng, Y. B. Jun, and X. Zhao, "Soft semirings," Computers and Mathematics with Applications, vol. 56, no. 10, pp. 2621-2628, 2008.

[15] G. Selvachandran and A. R. Salleh, "Introduction to vague soft groups," submitted.

[16] T. Vougiouklis, "A new class of hyperstructures," Journal of Combination and Information Systems and Sciences, vol. 20, pp. 229-235, 1995.

[17] V. Leoreanu-Fotea, F. Feng, and J. Zhan, "Fuzzy soft hypergroups," International Journal of Computer Mathematics, vol. 89, no. 8, pp. 963-974, 2012.

[18] P. Corsini, Prolegomena of Hypergroup Theory, Aviani, Tricesimo UD, Italy, 2nd edition, 1993. 

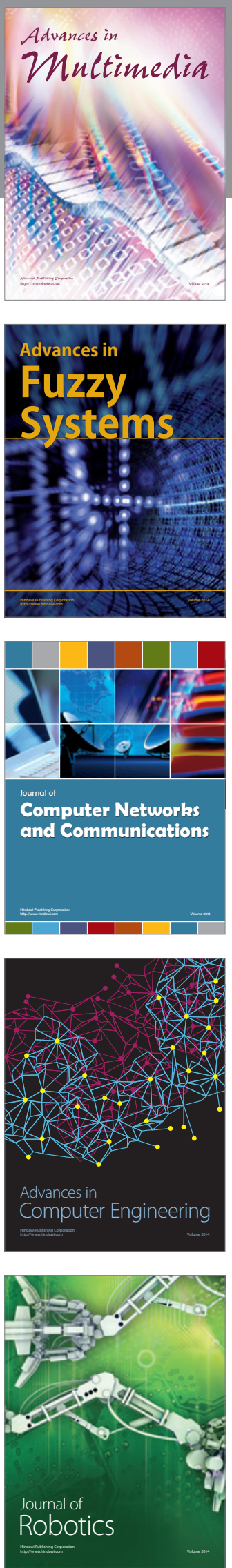

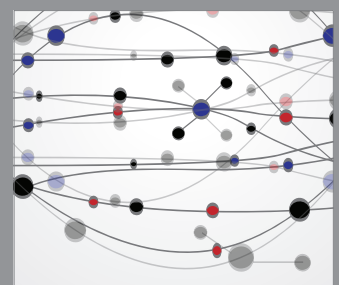

The Scientific World Journal
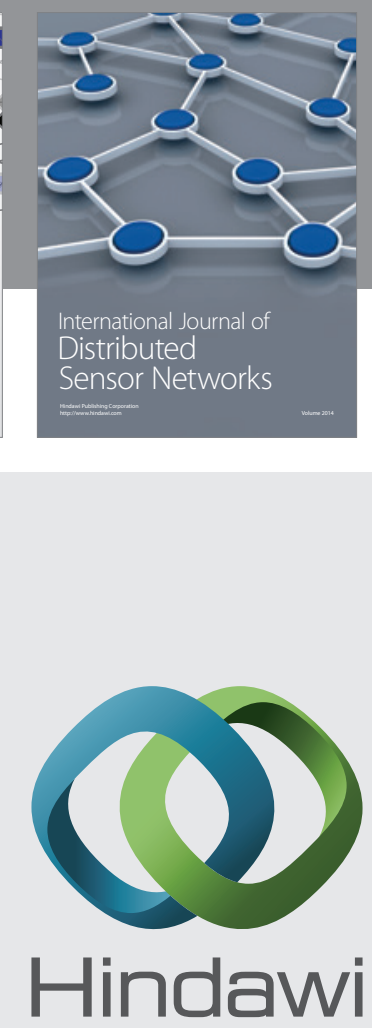

Submit your manuscripts at

http://www.hindawi.com
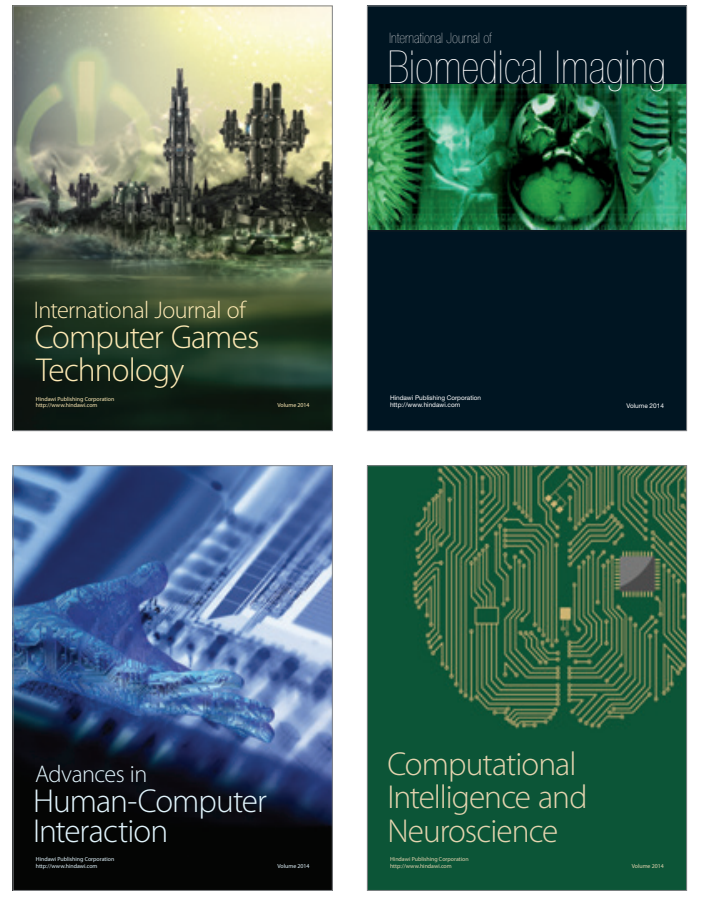
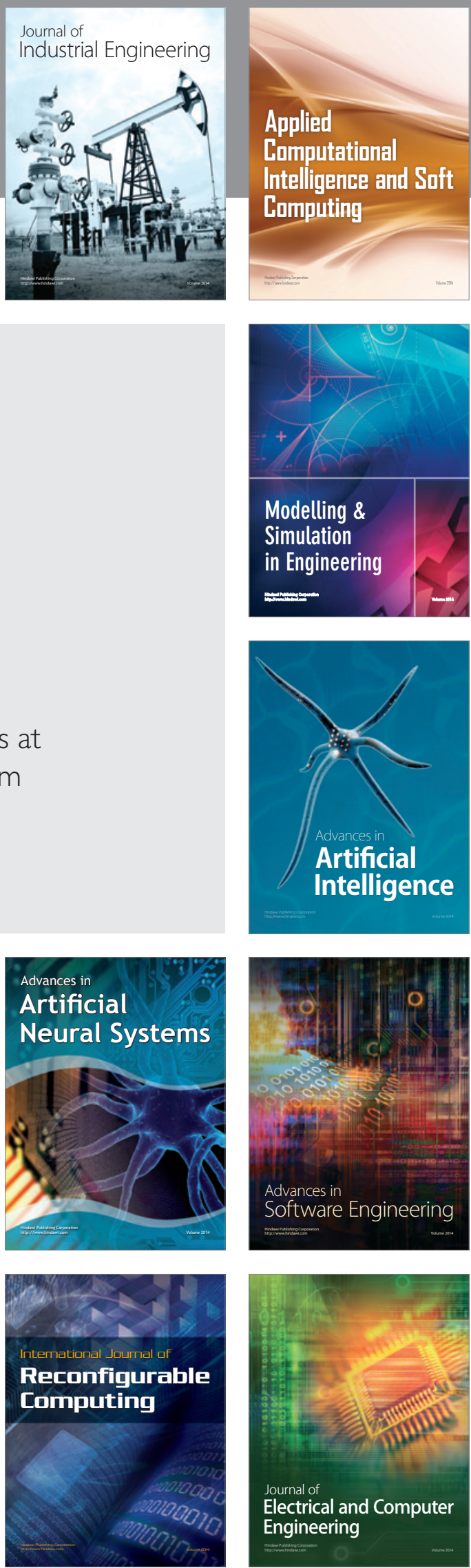